\title{
Evaluation of Drug Dosing in Renal Failure
}

\author{
Mohamed A. Hammad ${ }^{1}$, Ahmed A. Khamis ${ }^{1}$, Khaled M. Al- Akhali ${ }^{2}$, Tarek M. \\ Elsayed $^{3}$, Ashwag M. Alasmri ${ }^{4}$, Eman M. Al-Ahmari ${ }^{4}$, Enas M. Mossa ${ }^{4}$, Nada \\ M. Al-Gahtani ${ }^{4}$, Yasmin El-Sobky ${ }^{5}$ \\ 1 Department of Clinical Pharmacy, School of Pharmacy, Universiti Sains Malaysia, Malaysia \\ 2 Department of Clinical Pharmacy, School of Pharmacy, King Khalid University, Kingdom of Saudi Arabia \\ 3 Department of Pharmacy Practice, Kulliyyah of Pharmacy, International Islamic University Malaysia \\ 4 Aseer Central Hospital, Ministry of Health, Abha, Kingdom of Saudi Arabia \\ 5 Department of Biostatistics, High Institute of Public Health, Alexandria University, Egypt
}

\begin{abstract}
:
Introduction: Rapid accumulation and toxicity can be developed if drugs dosages are not adjusted in patients with chronic kidney diseases (CKD).

Objectives: To evaluate the prevalence of correct dosing in chronic kidney diseases depending on renal function estimation.

Study Design: A cross-sectional observational study involving patients in medical ward from January to July 2014 at Aseer Central Hospital in Abha, Kingdom of Saudi Arabia

Methods: Demographic data were collected from patients, patients' files and healthcare providers. Dosage adjustment depends on the patients' kidney function, most often estimated by the patient's glomerular filtration rate (eGFR) calculated by the CKD-EPI (Chronic Kidney Disease Epidemiology Collaboration) and a computerized system was used to determine the adjusted renal dose.

Results: From total 2470 prescribed drugs for 362 inpatients, $420(17 \%)$ drugs need a dosing adjustment in chronic renal failure and $2050(83 \%)$ drugs do not need. From the 420 drugs that need a dosing adjustment, the dose of $202(48.1 \%)$ drugs were adjusted, and of 218 (51.9\%) drugs were not. Drugs that need a readjustment are allopurinol, ampicillin, cefepime, cefixime, ceftazidime, ceftriaxone, gentamicin, levofloxacin, meropenem, metoclopramide, metronidazole, ranitidine, rosuvastatin, tazocin, tienam and vancomycin.

Conclusions: The dosing of more than half of the medications that need dosing adjustment in CKD were not adjusted which can increase the side effects and toxicity of these drugs for CKD patients. It is the pharmacist duty by co-operation with the medical team to ensure the prescribing of optimal dose.

Keywords: Aseer Central Hospital, Chronic kidney disease, chronic renal failure, $\mathrm{CrCl}$, glomerular filtration rate, dialysis, dosing adjustment, Renal dysfunction, Renal impairment.

\section{Impact on practice}

- $\quad$ More focusing on prescribing is needed for drugs dose adjustment in patients with CKD.

- Antibiotics constitute the majority of medications that need dosing adjustment in CKD.

- Using computerized renal dosage adjustment program by pharmacists, clinicians, and general physician will result in appropriate drugs dosage and decrease the chance of adverse drug reactions and hospitalization rate and cost.

- The study gives a flashlight on the importance of the collaboration among the medical team to improve the patients' outcome and quality of life.
\end{abstract}

\section{Introduction}

Chronic kidney disease is a common, progressive disease that is becoming a universal public health problem [1]. The guidelines define chronic kidney diseases (CKD) as either renal impairment or a reduced glomerular filtration rate $(\mathrm{GFR})<60 \mathrm{~mL} / \mathrm{min} / 1.73 \mathrm{~m}^{2}$ for at least three months. Whatever the causing etiology, once the loss of nephrons and decrease of functional renal mass reaches a particular level, the remaining nephrons start a process of irreversible sclerosis that results in a progressive reduction in the GFR [2, 3]. CKD is more prevalent in the elderly than the younger population. However, while younger patients with CKD typically experience progressive renal damage, $30 \%$ of patients $>65$ years of age with chronic renal diseases have stable disease [4]. In patients with renal dysfunction, the renal excretion of such drugs and their metabolites is impaired, leading to their accumulation in the body if no dose adjustment is made. Also, plasma protein binding of medicines may show a significant decline, which in turn could affect the pharmacokinetic processes of distribution and elimination. The activity of many drug-metabolizing enzymes and drug transporters has been demonstrated to be impaired in chronic renal failure. In patients with the end-stage renal disease, dialysis methods such as 
hemodialysis and continuous ambulatory peritoneal dialysis may remove medications from the body, necessitating dosage adjustment. Inappropriate dosing in patients with renal dysfunction can cause toxicity or ineffective therapy. Therefore, the standard dosage regimen of a drug may have to be adjusted in a patient with renal dysfunction [5].

Also, many drugs that are not dependent on the kidneys for elimination may exert untoward effects in the uremic milieu of advanced renal dysfunction. A familiarity with basic pharmacologic rules and a systematic approach are necessary when readjusting drug dosages in patients with renal impairment [6]. The distinct steps involve estimating the patient's creatinine clearance, selecting and administering a loading dose, designing a maintenance dose, and a decision regarding measuring of drug concentrations. If dosage adjustment was made correctly, treatment in patients with chronic kidney diseases should achieve the desired pharmacologic effects while avoiding drug toxicity. Physicians should not oversimplify the pharmacologic complexities presented by patients with renal failure by relying excessively on nomograms and "cookbook" equations. In addition to renal dysfunction, patients with renal disease often have alterations in pharmacokinetics such as bioavailability, protein binding, hepatic metabolism, and volume of distribution. An awareness of biologically active or toxic metabolites of parent compounds that increase when the kidney function is reduced, also necessary to avoid toxicity. The effects of dialysis on drug elimination and the need for supplemental dosing are additional considerations in patients undergoing renal replacement therapy [7].

Patients with renal dysfunction and those on hemodialysis commonly suffering from more drug-related problems [8]. Such patients are at greater risk as they need complex therapeutic regimens with five or more medications and twelve or more medication doses per day that require a more check up and dosage amendment [9], and they usually have other concurrent diseases including diabetes mellitus, hypertension, coronary artery diseases, and infections $[10,11]$. They are mostly nonadherent with treatment. Many studies have established that the incidence of adverse drug events is much higher in patients with renal impairment than those without renal insufficiency $[12,13]$.

Also, drug-related problems can lead to an increase in morbidity, mortality, and the cost of healthcare. Misuse of medications can increase adverse drug effects that may be reflected by excessive length of hospital stay [14], and excessive health care utilization and cost [15]. Significant numbers of adverse drug events are predictable and often preventable [16]. Preventable adverse drug reactions are usually a result of medication errors $[17,18]$. Prescribing errors often, occur because the prescribers do not have immediate access to all the needed data related to the drugs or the patient to adjust drug dosage [19].

\section{Aims of the study}

To evaluate the prevalence of adherence for correct dosing in chronic renal diseases and dialysis depending on renal function estimation.

\section{Methods}

A cross-sectional observational study was conducted to collect data from patients, patients' files and health provider team as shown in (Fig. 3.1). Patient's glomerular filtration rate (GFR) was calculated by the CKDEPI (Chronic Kidney Disease Epidemiology Collaboration) formula [20, 21]. CKD progression was defined based on decline in GFR category (>90 [Stage 1 (S1)], 60-89 [S2], 30-59 [S3], 15-29 [S4], <15 [S5] ml/min/1.73 m²) [22]. A computerized system was used to determine the renal dose of prescribed medicines. Five hundred patients were assessed for renal function and dosing adjustments. A stepwise approach to adjust drug dosages for patients with renal insufficiency as demonstrated in (Table 3.1) [23]. Patients have serum creatinine test and with age 18 years and above were included in the study. While patient does not have renal function test, patients under 18 years old, pregnant, patients with HIV and cancer diseases were excluded. Statistical analysis made by Excel program and SPSS 22.00. In this investigation, continuous variables were presented as mean $( \pm \mathrm{SD})$, and categorical variables were reported as counts and percentages. 


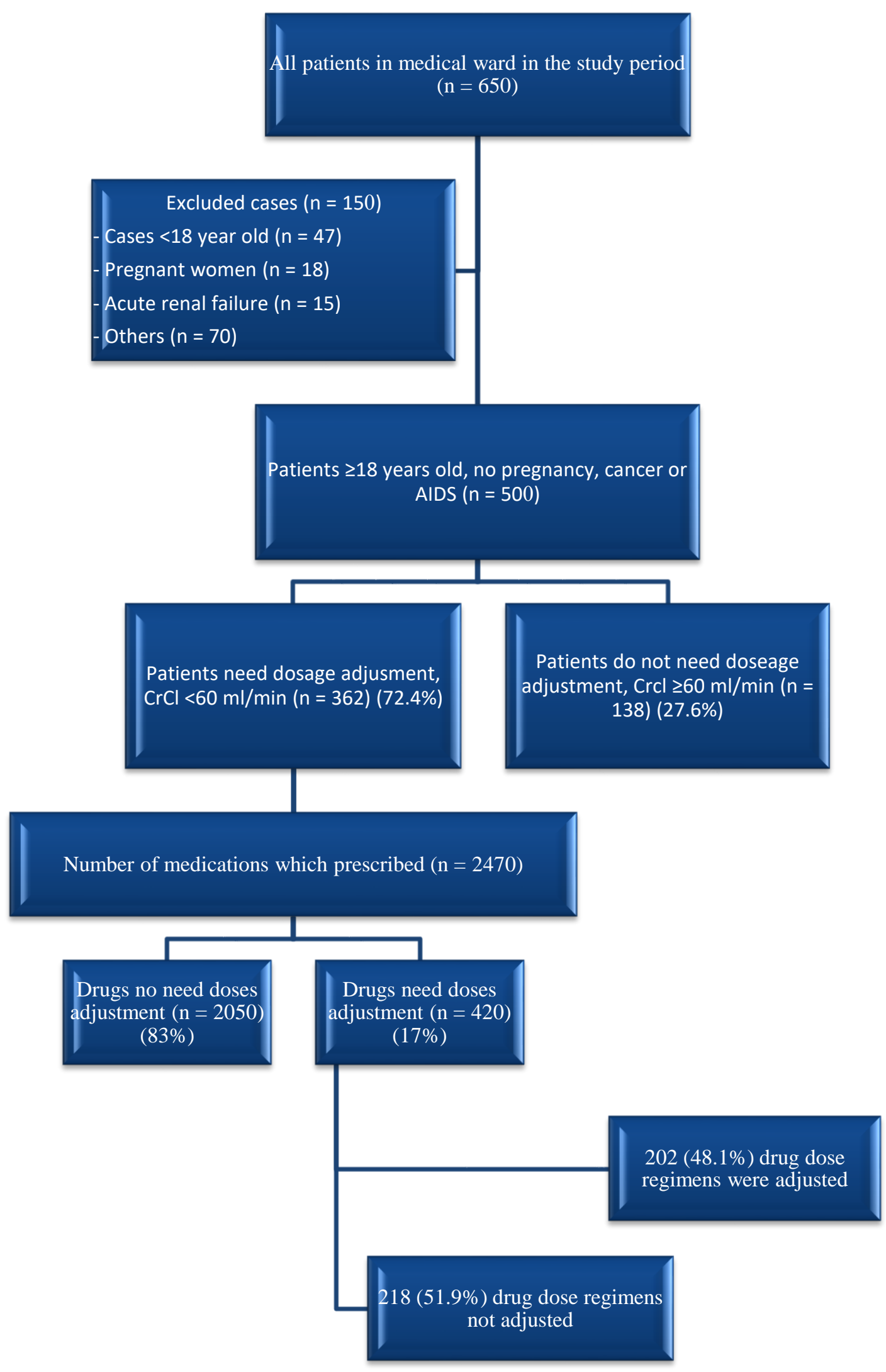

Figure 3.1. The flow chart of the study, AIDS: Acquired immune deficiency syndrome 
Table 3.1. A stepwise approach to adjust drug dosages for patients with renal insufficiency [24]

\begin{tabular}{|l|l|l|}
\hline Step & Task & Duty \\
\hline Step 1 & $\begin{array}{l}\text { Obtain history and relevant } \\
\text { demographic/ clinical } \\
\text { information }\end{array}$ & $\begin{array}{l}\text { Record demographic information, obtain past medical history } \\
\text { including history of renal disease and record current laboratory } \\
\text { information (e.g., serum creatinine) }\end{array}$ \\
\hline Step 2 & Estimate creatinine clearance & $\begin{array}{l}\text { Use the CKD-EPI formula for creatinine clearance calculation, } \\
\text { and a computerized system to estimate creatinine clearance, or } \\
\text { calculate creatinine clearance from timed urine collection }\end{array}$ \\
\hline Step 3 & Review current medications & $\begin{array}{l}\text { Identify drugs for which individualization of the treatment } \\
\text { regimen will be necessary }\end{array}$ \\
\hline Step 4 & $\begin{array}{l}\text { Calculate individualized } \\
\text { treatment regimen }\end{array}$ & $\begin{array}{l}\text { Determine treatment goals; calculate dosage regimen based on } \\
\text { pharmacokinetic characteristics of the drug and the patient's } \\
\text { renal function }\end{array}$ \\
\hline Step 5 & Monitor & $\begin{array}{l}\text { Follow up parameters of drug response and toxicity; monitor } \\
\text { drug levels if available/ applicable }\end{array}$ \\
\hline Step 6 & Revise regimen & $\begin{array}{l}\text { Adjust regimen based on drug response or change in patient } \\
\text { status (including renal function) as warranted }\end{array}$ \\
\hline
\end{tabular}

\subsection{Demographic data}

\section{Results}

Five hundred patients were included in the project; with a mean age of $62.5 \pm 16.5$ years. More than half of patients were female, $325(65 \%)$. The mean BMI was $26 \pm 1.15 \mathrm{~kg} / \mathrm{m}^{2}$. The majority of patients were having normal weight $270(54 \%), 75(43 \%)$ patients had overweight, and obesity and only $10(2 \%)$ patients were underweight. While $170(34 \%)$ patients were smokers, $100(20 \%)$ were ex-smokers and $230(46 \%)$ patients were non-smokers.

\subsection{Comorbidities}

Patients in this study had multiple co-morbidities, with a mean number of $3.56 \pm 2.44$ per patient. The most common diseases among 500 patients with were hypertension 275 (55\%), diabetes mellitus 238 (47.6\%), dyslipidemia 205 (41\%), and ischemic heart disease 150 (30\%), detailed are given in (Fig.4.1.).

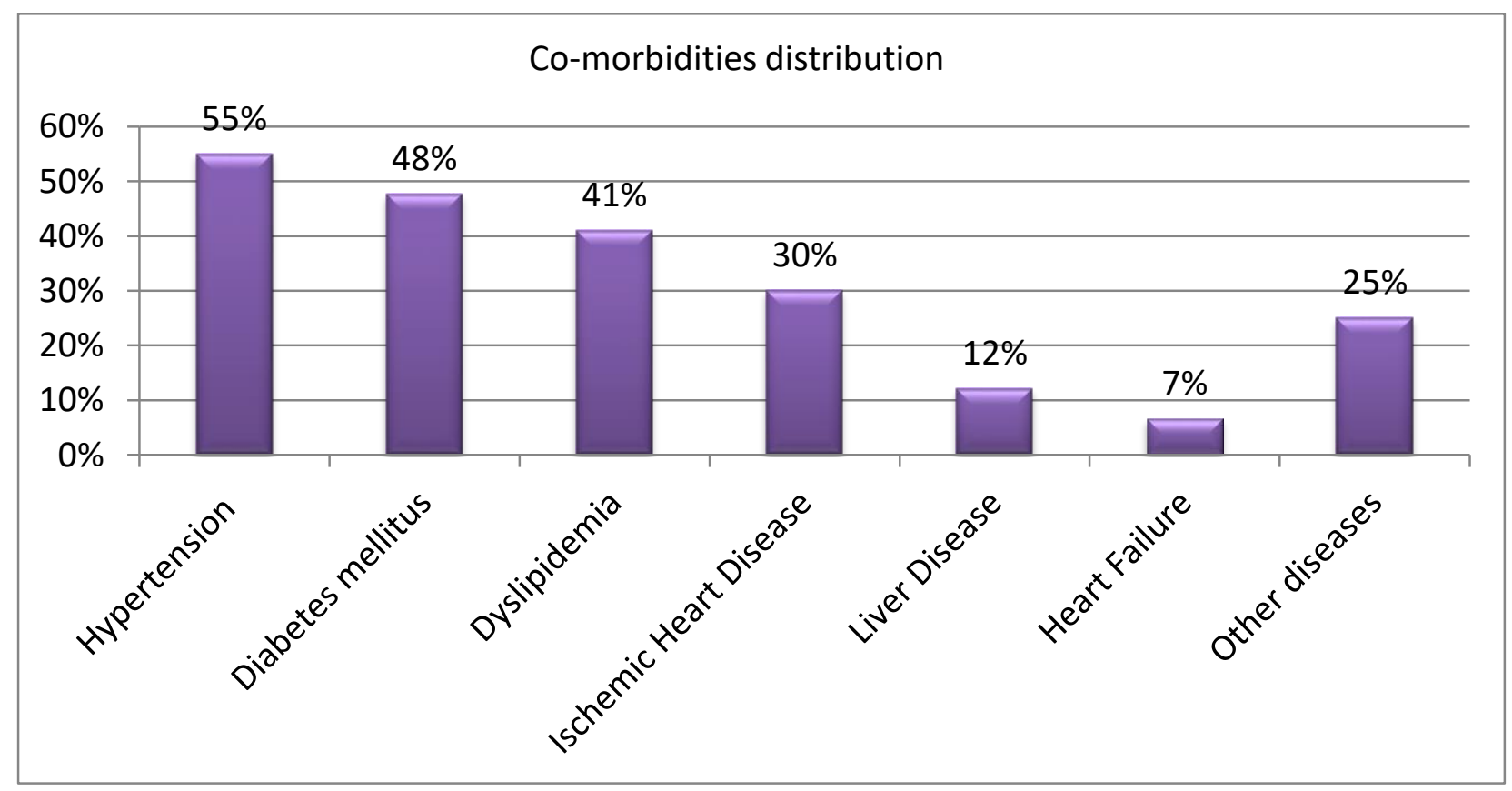

Figure 4. 1. Co-morbidities frequency distribution of studied patients

\subsection{Chronic renal failure distribution according to creatinine clearance}

Patients in this study have a different degree of chronic renal failure. More than half of them have stage 4 or end-stage renal disease as demonstrated in (Fig. 4.2.) 


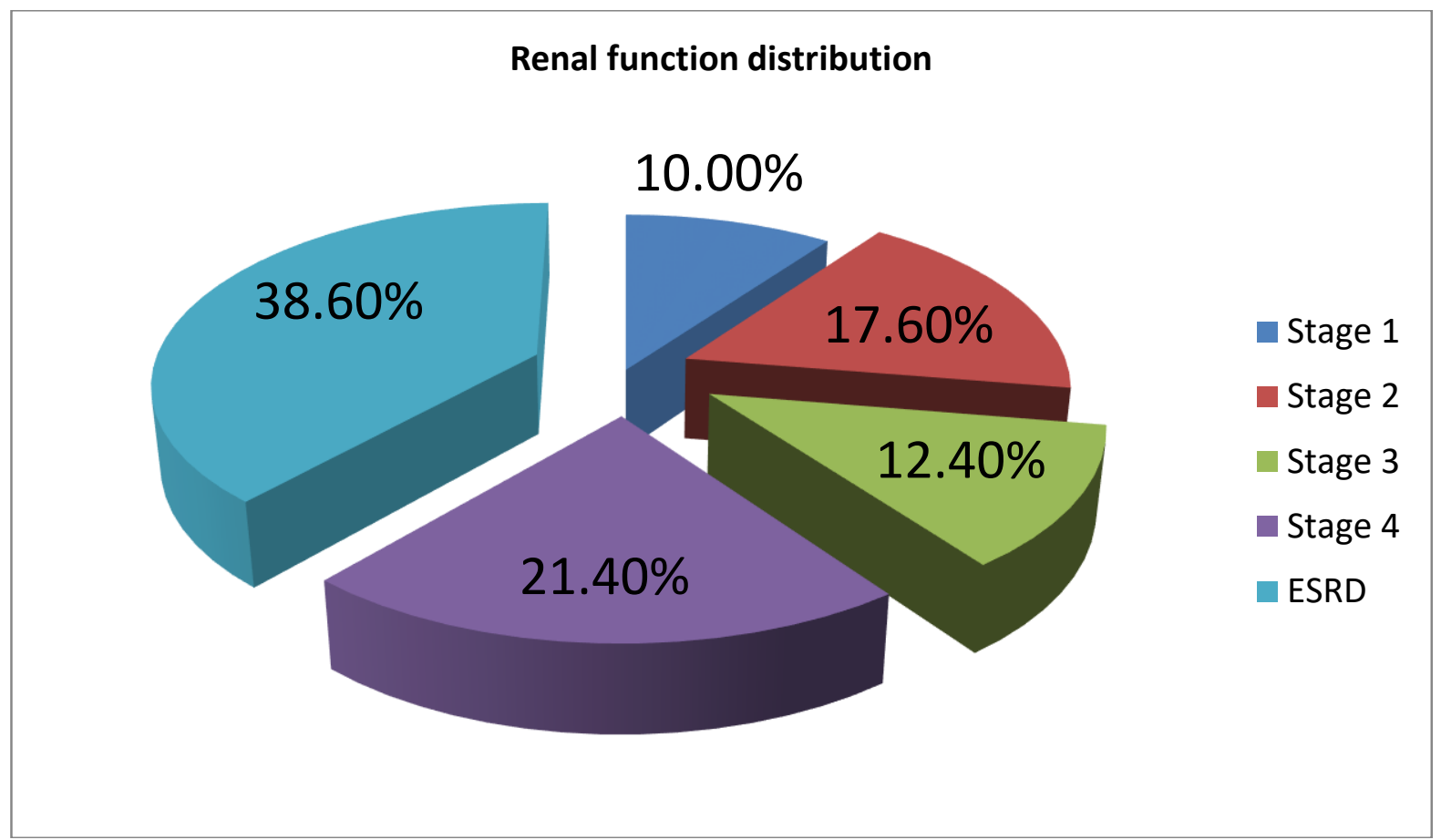

Figure 4.2. Chronic renal failure distribution according to creatinine clearance, $\mathrm{n}=(500)$

\subsection{Medications}

A total of 2470 medications were reviewed. Patients in this study were often prescribed multiple medications, with a mean number of $6.8 \pm 4.1$ per patient. The most commonly prescribed medicines are antibiotics (15.9\%), followed by vitamins and minerals supplements (12.1\%), drugs for peptic ulcer prophylaxis and treatment $(9.9 \%)$. More details are given in (Table 4.1).

Table 4.1. The frequency distribution of various drugs used by studied patients, $\mathrm{n}=2470$

\begin{tabular}{|l|l|l|l|}
\hline Variable & No. (\%) & Variable & No. (\%) \\
\hline Antibiotic & $393(15.9 \%)$ & B-blockers and CBs & $146(5.9 \%)$ \\
\hline Vitamins and minerals & $299(12.1 \%)$ & Diuretic & $124(5.9 \%)$ \\
\hline Other drugs & $299(12.1 \%)$ & ACEIs and ARBs & $126(5.1 \%)$ \\
\hline Anti-peptic ulcer & $245(9.9 \%)$ & Anti-platelets & $82(3.3 \%)$ \\
\hline Analgesic & $200(8.1 \%)$ & Bronchodilator & $82(3.3 \%)$ \\
\hline Antidiabetics & $173(7 \%)$ & Laxative & $64(2.6 \%)$ \\
\hline Statins & $154(6.2 \%)$ & Antiemetic & $64(2.6 \%)$ \\
\hline
\end{tabular}

ACEIs: Angiotensin-converting-enzyme inhibitor, ARBs: Angiotensin II receptor blocker, CCBs: Calcium channels blockers

\subsection{Dose adjustment in chronic renal failure}

From a total of 2470 prescribed medicines $420(17 \%)$ medication needed dosing adjustment in CKD and 2050 (83\%) drugs did not need that (Fig.4.3). From the 420 drugs that need dosing adjustment the dosing of only $202(48.1 \%)$ medications were adjusted, (Fig. 4.4). Drugs that need dosing adjustment in this study are allopurinol, ampicillin, cefepime, cefixime, ceftazidime, ceftriaxone, gentamicin, levofloxacin, meropenem, metoclopramide, metronidazole, ranitidine, rosuvastatin, tazocin, tienam and vancomycin. 


\section{$17.00 \%$}

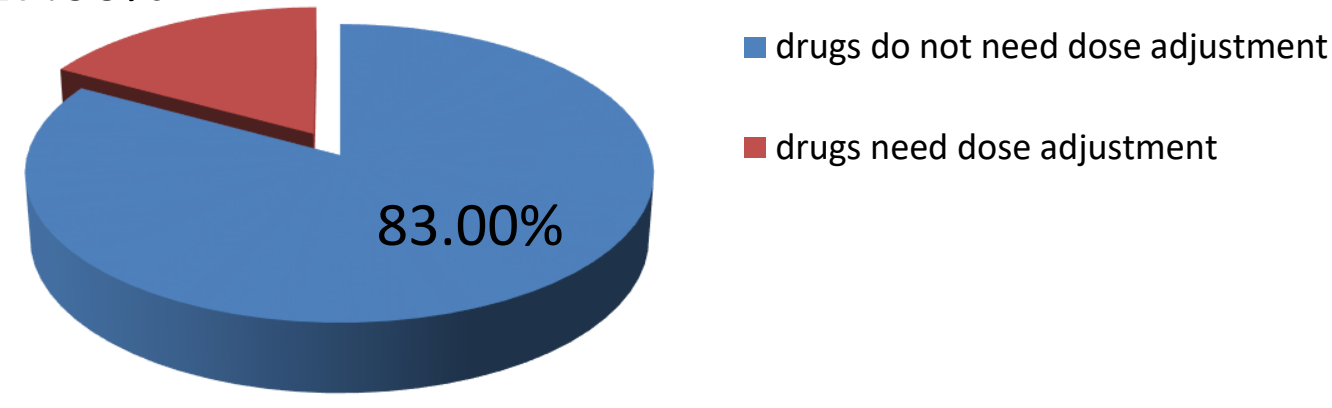

Figure 4.3. Distribution of 2470 drugs based on the need for dosing adjustment in the study sample

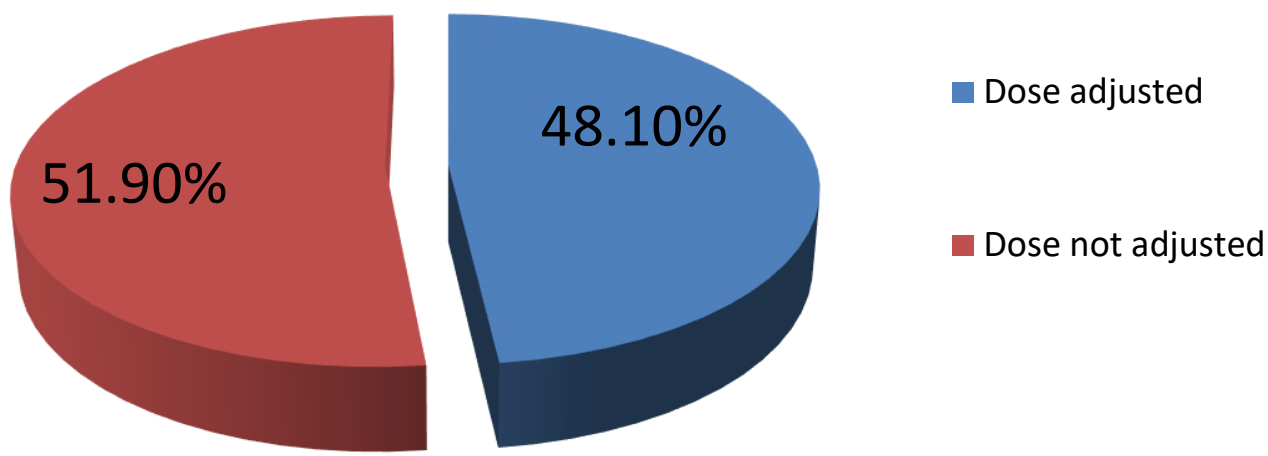

Figure 4.4. Dosing adjustment distribution among the 420 drugs that need dosing adjustment in CKD

\section{Discussion}

The design of the optimal dosage regimen for patients with CKD is dependent on the availability of an accurate characterization of the relationship between the pharmacokinetic parameters of the drug and renal function, and an accurate assessment of the patient's renal function. Before1998, there was no consensus regarding the explicit criteria for characterization of the relationship between the pharmacodynamics and pharmacokinetics of a drug and renal function. The United States Food and Drug Administration (FDA) industry guidance issued in May 1998 provided a framework to help companies decide when they should conduct such a "characterization" study and proposed clear recommendations for study design, data analysis, and assessment of the impact of the project results on drug dosing. Thus the quality of data available to clinicians has improved dramatically in the last ten years [25].

\subsection{Demographic data}

In this study 500 patients were included with a mean age of $62.5 \pm 16.5$ years. More than half of them were female, $325(65 \%)$ and $175(35 \%)$ were male. Our study tallies The Third National Health and Nutrition Examination Survey (NHANES III), a national investigation of more than 18,000 persons 20 years of age or older conducted from 1988 through 1994, provides information on the stages of CKD in the U.S. population. From these data, populations at increased risk for developing ESRD include the older population, particularly patients 
65 years of age and older. More than $49 \%$ of incident hemodialysis patients in 2004 were age 65 or older, a much larger percentage than for the peritoneal dialysis and transplant populations [26, 27].

\subsection{Co-morbidities}

Patients in this study had multiple co-morbidities, with a mean number of $3.56 \pm 2.44$ per patient. The most common diseases among them were hypertension 275 (55\%), diabetes mellitus 238 (47.6\%), dyslipidemia 205 (41\%), and ischemic heart disease 150 (30\%), (Fig. 4.1.). Chronic kidney disease most often results from a progressive loss of functioning nephrons caused by a primary kidney disease or as a secondary complication of certain systemic diseases, but it can also arise from an acute event causing irreversible damage to the kidneys. The leading causes of ESRD in patients newly diagnosed in the USA in 2004 were diabetes mellitus (44\%), hypertension (27\%), and chronic glomerulonephritis (8\%). Among Native Americans, diabetes is the predominant etiology, accounting for nearly two-thirds of the ESRD cases. Diabetes also accounts for higher rates of ESRD in blacks and Hispanics than in whites. Another of the Healthy People 2010 national health objectives for CKD is to focus on efforts to reduce the incidence of ESRD in blacks, Hispanics, and Native Americans. Hypertension remains the most common cause of ESRD among blacks, with approximately one-third of cases attributed to this disease $[28,29]$. A variety of causes are responsible for the remaining cases of ESRD, among which are cystic kidney disease, other urologic causes, and acquired immunodeficiency syndrome (AIDS) nephropathy [30, 31].

\subsection{Chronic renal failure distribution according to creatinine clearance}

In our findings majority of patients have ESRD 193 (38.6\%) and stage 4 was 107 (21.4\%), (Fig. 4.2.). Our study tally with data describing the ESRD population are made available annually by the U.S. Renal Data System (USRDS). The reports characterize the development, treatment, morbidity, and mortality associated with ESRD in the United States and include data from patients with kidney transplants. Based on the most recent data from the USRDS, more than 472,000 patients were receiving renal replacement therapy for ESRD at the end of 2004, with approximately 104,000 new patients starting treatment during that year. A continued increase in incidence rates of ESRD has been observed over the past decade, although this rate has decreased to approximately $1.5 \%$ to $5 \%$ per year over the previous five years. The continued increase in incidence rates of ESRD, in conjunction with a relatively stable death rate for this population, accounts for the overall increase in the prevalence of ESRD. CKD has been identified as one of the focus areas for the Healthy People 2010 national health initiative; one of the specific objectives is to reduce the number of new cases of ESRD [32].

\subsection{Dose adjustment in chronic renal failure}

In this study from total 2470 prescribed medicines 420 (17\%), medications need a dose adjustment, and $2050(83 \%)$ drugs do not require dosage regimen change in chronic renal failure as demonstrated in (Fig.4.3). From 420 drugs need dose adjustment 202 (48.1\%) medications were adjusted, and 218 (51.9\%) medicines were not adjusted as described in (Fig. 4.4). The drugs need a readjustment in our study are allopurinol, ampicillin, cefepime, cefixime, ceftazidime, ceftriaxone, gentamicin, levofloxacin, meropenem, metoclopramide, metronidazole, ranitidine, rosuvastatin, tazocin, tienam and vancomycin.Significance overestimation of $\mathrm{CrCl}$ calculations may occur in people with reduced muscle mass (e.g. elderly, paralysis). The clinical pharmacist should use their clinical judgment regarding these changes and communicate their recommendations with the medical team as appropriate [33, 34].

\subsubsection{Allopurinol}

Renal elimination of allopurinol metabolites decreases in kidney deficiency. The accumulation of these active metabolites may result in harmful effects if the dosage is not modified [35, 36]. For most elderly, a maintenance dose of $100 \mathrm{mg} /$ day is sufficient [37]. Loading dose of $100 \mathrm{mg}$ on alternate days is recommended for patients with a GFR $<10 \mathrm{~mL} / \mathrm{min}$, [38] or if possible, the medicine should be avoided altogether in this situation [39]. Also, Randall Faull (2007) stated that; Allopurinol is used in the treatment of gout to decrease urinary and serum uric acid concentrations. As allopurinol, and its active main metabolite oxypurinol, are mainly eliminated in the urine, they accumulate in patients with kidney impairment so the dose should be reduced. The manufacturers recommend initiate therapy with a maximum dose of $100 \mathrm{mg} /$ day and raising it only if the urinary or serum urate is not satisfactorily controlled [40].

\subsubsection{Ampicillin, amoxicillin, and cephalosporins (Cefepime, cefixime, ceftazidime and ceftriaxone)}

The standard adult dose is one $500 \mathrm{mg}$ tablet of amoxicillin every two times daily or one $250 \mathrm{mg}$ tablet of amoxicillin three times daily. For infections of the respiratory system and more severe infections, the dose should be one $875 \mathrm{mg}$ tablet of amoxicillin every 12 hours or one $500 \mathrm{mg}$ tablet of amoxicillin every 8 hours. Patients with impaired renal function do not require a reduction in dose unless the renal dysfunction is serious [41]. Severely impaired patients with eGFR $<30 \mathrm{~mL} / \mathrm{min}$. should not receive the $875 \mathrm{mg}$ tablet. Patients with $\mathrm{CrCl}$ 
of 10 to $30 \mathrm{~mL} / \mathrm{min}$. should give $500 \mathrm{mg}$ or $250 \mathrm{mg}$ every 12 hours, depending on the severity of the infection. Patients with a glomerular filtration less than $10 \mathrm{~mL} / \mathrm{min}$., should receive $500 \mathrm{mg}$ or $250 \mathrm{mg}$ every 24 hours, based on the seriousness of the disease. End-stage renal failure (ESRF) on hemodialysis: 250 to $500 \mathrm{mg}$ amoxicillin every 24 hours; administer dose both during and after dialysis. Do not give extended-release or $875 \mathrm{mg}$ tablet [42, 43].

Ceftazidime is a cephalosporin that has excellent activity against most strains of Pseudomonas sp. As with most cephalosporins, ceftazidime primarily is cleared by the kidneys, with little non-renal or hepatic elimination. Because drug clearance is approximately one-third of normal, patient would require about one-third of the usual daily dose (i.e., $1 \mathrm{~g}$ every 24 hours). As with other cephalosporins, ceftazidime has a large therapeutic window [44]. Failure to reduce the dose from a standard dose of $1 \mathrm{~g}$ every 8 hours might, however, lead to accumulation of ceftazidime, predisposing seizures and other adverse effects associated with toxic $\beta$-lactam antibiotic plasma levels [45]. Because only $21 \%$ of ceftazidime is protein bound and its $\mathrm{Vd}$ is $0.2 \mathrm{~L} / \mathrm{kg}$, it should be readily removed by hemodialysis. The mean dialysis clearance of ceftazidime is $55 \mathrm{~mL} /$ minute, with $55 \%$ of the drug removed during 4 hours of conventional hemodialysis. A supplemental dose of ceftazidime should be given following each hemodialysis session to maintain a therapeutic concentration. Half of the daily ceftazidime dose should be administered after each dialysis session [46].

\subsubsection{Aminoglycosides}

Aminoglycosides are almost eliminated by the kidneys; thus, the clearance of these drugs essentially is equal to the glomerular filtration rate (GFR). The pharmacokinetic properties of gentamicin and tobramycin are similar. A close correlation exists between $\mathrm{CrCl}$ and gentamicin total body clearance. As renal function deteriorates, aminoglycoside doses must be modified to maintain the desired peak and trough plasma concentrations. Failure to appropriately adjust the dosage of aminoglycosides in renal insufficiency can lead to high drug plasma levels that can result in ototoxicity and nephrotoxicity. In many cases, the aminoglycoside dose can be modified by extending the dosing interval rather than just reducing the dose. This extending permits maintenance of sufficient peak plasma concentrations to ensure efficacy while allowing for sufficient elimination between doses to produce trough levels $<2 \mathrm{mg} / \mathrm{L}$. Gentamicin has a molecular weight of about 500 and is significantly removed by conventional hemodialysis [47]. Gentamicin has a relatively small Vd, averaging 0.25 $\mathrm{L} / \mathrm{kg}$, and is about $10 \%$ bound to proteins. The dialysis clearance of gentamicin using conventional methods depends on the actual filter used as well as the blood and dialysate flow rates. Dialysis clearance averages 45 $\mathrm{mL} /$ minute compared with a mean plasma clearance of $5 \mathrm{~mL} /$ minute in patients with end-stage renal disease (ESRD) [48]. Therefore, gentamicin dose must be adjusted to compensate for the amount of drug that will be removed by dialysis, because drug removal represents a combination of drug elimination by the body and dialysis [49].

\subsubsection{Metoclopramide}

Since metoclopramide is eliminated mainly through the kidneys, and the risk of toxic reactions to this drug may be higher in patients with renal insufficiency. When $\mathrm{CrCl}<50 \mathrm{ml} / \mathrm{min}$, give $75 \%$ of normal metoclopramide. In those patients whose $\mathrm{CrCl}$ is lower than $40 \mathrm{~mL} / \mathrm{min}$, treatment should be started at approximately the half of the standard dosage [50]. According to clinical efficacy and safety considerations, the dosage may be increased or decreased as appropriate. Not dialyzable ( $0 \%$ to $5 \%$ ); supplemental dosing is not necessary [51].

\subsubsection{Metronidazole}

The adult dose is $500 \mathrm{mg} \mathrm{PO} / \mathrm{IV}$ q8h. Same for adult and pediatric, $\mathrm{CrCl}<10$ or severe hepatic dysfunction: consider $50 \%$ at same interval if $>14$ days' duration [52, 53]. Intermittent hemodialysis (IHD) (give metronidazole after hemodialysis on dialysis days): Dialyzable (50\% to $100 \%)$ : a dose of $0.5 \mathrm{~g}$ every three or two times daily. Dosing regimen highly dependent on clinical indication (C. difficile colitis vs. trichomoniasis) [54]. Dosing reliant on the assumption of thrice weekly, complete IHD sessions. Continuous renal replacement therapy (CRRT) [55]. Medicine elimination is highly dependent on the method of renal replacement, the rate of flow and type of filter. Proper dosing needs close monitoring of pharmacologic effect, signs of adverse reactions due to drug accumulation, as well as drug concentrations about target trough (if applicable) [56, 57].

\subsubsection{Ranitidine}

The pharmacokinetic behavior of a single oral $150 \mathrm{mg}$ ranitidine dose was investigated in six subjects with severe chronic renal failure (CRF) $(\mathrm{CrCl} 2-18 \mathrm{ml} / \mathrm{min})$ and compared to that in ten patients with duodenal ulceration but the normal renal function $(\mathrm{N})(\mathrm{CrCl} 69-125 \mathrm{ml} / \mathrm{min})$. Although the maximum concentrations (Cmax) were significantly larger in CRF group when compared to $\mathrm{N}$ group $(P<0.025)$ there was no variation in the time taken to reach Cmax (Tmax). The area under the curve (AUC) was also significantly higher in the CRF 
group $(P<0.001)$ than in the $\mathrm{N}$ group. Within the $\mathrm{CRF}$ group, there was a significant variation in $\mathrm{Cmax}(\mathrm{CV}=$ $38 \%)$ and area under the curve $(46 \%)$ which may reflect different ranitidine bioavailability. The terminal elimination rate constant (beta) was significantly smaller $(P<0.001)$ in CRF group when compared with normal renal function group resulting in a median half-life for the chronic renal failure cohort of $7.3 \mathrm{~h}, 2.4$ times that of $\mathrm{N}$ cohort. The recovery of unchanged ranitidine in the urine was significantly less in renal impairment group $(P$ $<0.001)$ despite a considerable variation among interindividual in both groups. A significant linear relationship between $\mathrm{CrCl}$ and beta was shown $(\mathrm{r}=0.81 p<0.001)$. The results explain that ranitidine elimination is appreciably decreased in renal insufficiency. It is tentatively recommended that the standard $150 \mathrm{mg}$ dose should be halved while keeping the dosing interval unchanged at twelve hours in patients with severe renal impairment (creatinine clearance less than $30 \mathrm{ml} / \mathrm{min}$ ) [58].

\subsubsection{Rosuvastatin}

According to the manufacturer, rosuvastatin is contraindicated with severe renal impairment (RI) (creatinine clearance $<30 \mathrm{~mL} / \mathrm{min}$ ), but no dose adjustment is necessary for patients with mild to moderate RI. The recommended starting dose is $5 \mathrm{mg}$ in patients with moderate $\mathrm{RI}(\mathrm{CrCl}$ of $<60 \mathrm{~mL} / \mathrm{min})$. The dose of $40 \mathrm{mg}$ is contraindicated in patients with moderate RI. In a study in subjects with varying degrees of RI mild to moderate kidney dysfunction had no effect on plasma concentration of rosuvastatin or the N-desmethyl metabolite. Subjects with severe impairment (creatinine clearance $<30 \mathrm{~mL} / \mathrm{min}$ ) had a 3 -fold duplication in plasma concentration and a 9-fold enhancement in the $\mathrm{N}$-desmethyl metabolite concentration compared to healthy volunteers [59]. The RDH advises 5 to $20 \mathrm{mg}$ in all degrees of RI. Molitchrecommends avoiding doses $>10 \mathrm{mg}$ in patients with a GFR $<30 \mathrm{~mL} / \mathrm{min} / 1.73 \mathrm{~m}^{2}$ and suggests 10 to $40 \mathrm{mg}$ daily in stage 1 to $2 \mathrm{CKD}, 10$ to $20 \mathrm{mg}$ daily in stage $3 \mathrm{CKD}$, and 5 to $10 \mathrm{mg}$ daily in stage 4 to 5 CKD [60].

\subsubsection{Tazocin (Piperacillin and tazobactam)}

Piperacillin is anti-pseudomonal penicillin that is often used with an aminoglycoside to treat severe infections caused by gram-negative organisms [61]. Piperacillin is commonly given as a combination with tazobactam, a $\beta$-lactamase inhibitor [62]. In patients with normal renal function, piperacillin is primarily excreted unchanged by the kidney with a clearance of $2.6 \mathrm{~mL} /$ minute $/ \mathrm{kg}$, and a half-life of approximately one hour [63]. Doses of piperacillin/tazobactam can be as high as $4.5 \mathrm{~g}$ every 6 hours for the treatment of acute Pseudomonal sp. infections. In patients with ESRD, mean of piperacillin clearance and half-life values are $0.7 \mathrm{~mL} / \mathrm{minute} / \mathrm{kg}$ and 3.3 hours, respectively [64]. Although these parameters are significantly different, they are less than those expected for a drug primarily cleared by the kidneys, suggesting that some other compensatory mechanism for elimination must be present. Piperacillin is partially cleared by biliary excretion, a route of elimination that is increased in patients with renal failure $[65,66]$.

\subsubsection{Vancomycin}

Vancomycin is a bactericidal antibiotic with excellent activity against most gram-positive organisms such as methicillin-resistant Staphylococcus aureus (MRSA) and Streptococcus sp., including some isolates of Enterococcus faecalis. It is used empirically in the febrile neutropenic patient because the incidence of infection secondary to resistant organisms is much greater in this patient population. However, cases of vancomycinresistant enterococci (VRE) have emerged at rates as high as 50\%, raising concern and reducing its empiric use [67]. Vancomycin is poorly absorbed by the oral route and must be administered intravenously when used to treat systemic infections. As with many other antibiotics, vancomycin primarily is cleared by the kidneys [68]. Significant toxicities have been associated with elevated serum concentrations, making careful dosing modification in renal failure necessary [69]. Pharmacokinetic calculations can be used to individualize a dosing regimen to produce the desired peak and trough plasma levels. Unlike the aminoglycosides, the therapeutic range for vancomycin is less clear. Normally, doses are designed to achieve peak levels of 25 to $40 \mathrm{mg} / \mathrm{L}$ and trough levels of 10 to $15 \mathrm{mg} / \mathrm{L}$ [70]. The correlation between vancomycin toxicity (e.g., ototoxicity) and plasma levels is not well defined. Some clinicians have suggested, however, that plasma levels $\geq 80 \mathrm{mg} / \mathrm{L}$ may correlate with auditory dysfunction [71]. Vancomycin has an elimination half-life of 3 to 9 hours in patients with normal renal function; this increases to 129 to 189 hours in patients with ESRD [72].

\section{Limitation of Study}

There are a few potential limitations of our study was that: the scope of this study was limited to one hospital, and the reviewer of this study was not blinded to the study hypothesis or objectives. There is a need for more study to evaluate adverse outcomes of inappropriate dosage regimen. 


\section{Conclusion}

An important issue in drug therapy is dose adjustment in chronic kidney disease. Many drugs need to be adjusted depending on the person's kidney function. More than half of the medications that need dose adjustment were not amended which can increase the side effects and toxicity of these drugs for the patients. It is the pharmacist duty by co-operation with the medical team to ensure a patient is taking the optimal dose.

\section{Recommendation for pharmacist}

1. Identify patients at risk of CKD (e.g., Patients with diabetes, hypertension or glomerulonephritis).

2. Ask the patient about kidney function and examine the patient's lab report if accessible. If additional information is necessary, communicate the patient's physician.

3. Identify which medication may require dose adjustment in CKD.

4. Investigate medication profile for drug interactions which could affect serum level of renally excreted drugs and reduce renal function (e.g., nephrotoxic drugs).

5. Determine the dose adjustments needed for all medications, recommendations for dose adjustments based on renal function are provided in manufacturers' drug monographs, e-therapeutics+, lexicomp, Micromedex, etc.

6. Communicate recommendations for readjustments of the patient's medications to the physician.

7. Establish a monitoring plan for the patient because further dose adjustment may be required depending on patient's response.

\section{Acknowledgment}

Our special thanks to all staff of Aseer Central Hospital, especially the medical ward, department of pharmacy and laboratory staff for their kind support and help in facilitating our study.

\section{References}

[1]. Roger K. Verbeeck \&Flora T. Musuamba. Pharmacokinetics and dosage adjustment in patients with renal dysfunction, Eur J Clin Pharmacol (2009) 65:757-773

[2]. Waknine Y. Kidney Disease Classification to Include Albuminuria. Medscape Medical News. Available at http://www.medscape.com/viewarticle/776940. December 31, 2012; [Accessed at: July 24, 2016].

[3]. [Guideline] Kidney Disease: Improving Global Outcomes (KDIGO) CKD Work Group. KDIGO 2012 Clinical Practice Guideline for the Evaluation and Management of Chronic Kidney Disease. Kidney Int Suppl. 2013. 3:1-150.

[4]. O'Hare AM, Choi AI, Bertenthal D, Bacchetti P, Garg AX, Kaufman JS, et al. Age affects outcomes in chronic kidney disease. J Am Soc Nephrol. 2007 Oct. 18(10):2758-65. [Medline].

[5]. Roger K. Verbeeck\& Flora T. Musuamba, Pharmacokinetics and dosage adjustment in patients with renal dysfunction, Eur $J$ ClinPharmacol (2009) 65:757 - 773.

[6]. Gambertoglio JG. Effects of renal disease: altered pharmacokinetics In: Benet LZ et al., eds. Pharmacokinetic Basis for Drug Treatment New York: Raven Press; 1984.

[7]. Swan SK, Bennett WM: Drug dosing guidelines in patients with renal failure. West J Med 1992 Jun; 156:633-638.

[8]. Syed Sulaiman S. A., Mohamed Noor D. A., Mohamed A. Hammad, Khaled M. Al- Akhali, Ashwag M. Alasmri, Eman M. AlAhmari, Enas M. Mossa, Nada M. Al-Gahtani, Prospective Study of Evaluation of Antibiotics Dosage Adjustment in Patients with Chronic Renal Failure at Aseer Hospital. The 7th Asian Association of Schools of Pharmacy Conference, Taipei, Taiwan, JAASP 2015;1:142.

[9]. Mohamed A. Hammad et al, Prospective Study of Evaluation of Drug Dosage Adjustmentin Patient with Chronic Renal Failure at Aseer Hospital, The 10th Annual Scientific Research Day for Medical, Applied and Basic Sciences, King Khalid University, 5.05.2014, P92

[10]. Hammad, M. A., B. Tangiisuran, N. Abd El Aziz, and Y. Hassan. "A prospective study of uncontrolled glycaemia secondary to drugdrug interactions in type 2 diabetes mellitus patients at Penang general hospital in Malaysia.". In Pharmacotherapy, vol. 33, no. 5, pp. E50-E50.

[11]. Mohamed A. Hammad, Dzul Azri Mohamed Noor, Syed Azhar Syed Sulaiman, Nor Azizah Aziz, Yasmin Elsobky, A prospective study of prevalence of uncontrolled glycaemia in type 2 diabetes mellitus outpatients, American College of Clinical Pharmacy 2016, ACCP Virtual Poster Symposium, Pharmacotherapy, May 18-19, 2016.

[12]. Gabardi S, Abramson S. Drug dosing in chronic kidney disease. Med Clin North Am 2005;89:649-87.

[13]. Manley HJ, Cannella CA, Bailie GR, St Peter WL. Medication-related problems in ambulatory hemodialysis patients: A pooled analysis. Am J Kidney Dis 2005;46:669-80.

[14]. Bigler D et al. Prolonged respiratory depression caused by slow release morphine. Lancet 1984;1:1477.

[15]. Bonk ME, Krown H, Matuszewski K, Oinonen M. Potentially inappropriate medications in hospitalized senior patients. Am J Health Syst Pharm 2006;63:1161-5.

[16]. Saheb Sharif-Askari N, Syed Sulaiman SA, Saheb Sharif-Askari F, Al Sayed Hussain A, Adverse drug reaction-related hospitalisations among patients with heart failure at two hospitals in the United Arab Emirates, Int J Clin Pharm, Springer, 9- Dec.2014.

[17]. Ramesh M, Pandit J, Parthasarathi G. Adverse drug reactions in a south Indian hospital - their severity and cost involved. Pharmacoepidemiol Drug Safe 2003;12:687-92.

[18]. Van Dijk EA, Drabbe NR, Kruijtbosch M, De Smet P. Drug dosage adjustments according to renal function at hospital discharge. Ann Pharmacother 2006;40:1254-60.

[19]. Nightingale PG, Adu D, Richards NT, Peters M. Implementation of rules based computerized bedside prescribing and administration: intervention study. BMJ 2000;320:750-3.

[20]. Levey AS, Stevens LA, Schmid CH, et al. (May 2009). "A new equation to estimate glomerular filtration rate". Annals of Internal Medicine 150 (9): 604-12. 
[21]. Hougardy, JM; Delanaye, P; Le Moine, A; Nortier, J (2014). "Estimation of the glomerular filtration rate in 2014 by tests and equations: strengths and weaknesses.". Rev Med Brux. (in French). 35 (4): 250-7.

[22]. KDIGO 2012 Clinical Practice Guideline for the Evaluation and Management of Chronic Kidney Disease, Chapter 2: Definition, identification, and prediction of CKD progression, Kidney International Supplements (2013) 3, 5-14

[23]. Yahaya Hassan, Rowa'J Al-Ramahi, Noorizan Abd Aziz, Rozina Ghazali. Drug Use and Dosing in Chronic Kidney Disease, Annals Academy of Medicine, December 2009, Vol. 38 No. 12, p1095-1103

[24]. Gary R Matzke; George R Aronoff; Arthur J Atkinson Jr; William M Bennett; Brian S Decker; Kai-Uwe Eckardt, et al., Drug dosing consideration in patients with acute and chronic kidney disease - a clinical update from Kidney Disease: Improving Global Outcomes (KDIGO), Kidney International (2011) 80, 1122-1137.

[25]. Anonymous. Characterization of the relationship between the pharmacokinetics and pharmacodynamics of a drug and renal function. U.S. Department of Health and Human Services (http://www.fda.gov/ cber/guidelines.htm), FDA Guidance, May 1998.

[26]. U.S. Renal Data System, USRDS 2006 Annual Data Report Atlas of End-Stage Renal Disease in the United States, National Institutes of Health, National Institute of Diabetes and Digestive and Kidney Diseases, Bethesda, MD; 2006.

[27]. Alakhali KM, Selim M, Hammad MA. Evaluation of therapeutic drug monitoring of cyclosporine and tacrolimus in kidney transplant patients. JPCS.2014 Jan;3(8).

[28]. Khaled MA, AsifAnsari Md., Anwar HA Md. Analysis of Prevalence Risk Factor and Pharmacotherapy of Hypertension in Outpatients. Indian Journal of Pharmacy Practice. Volume 6 Issue 4 Oct - Dec, 2013, p: 64-66. Available at: https://www.researchgate.net/publication/270337790_Analysis_of_Prevalence_Risk_Factor_and_Pharmacotherapy_of_Hypertensio n_in_Outpatients?ev=prf_pub

[29]. Khaled M. Alakhali, Asif Ansari, Anwar HA, Analysis of Prevalence, Risk Factor and Pharmacotherapy of Hypertension in Outpatients. The 10th Annual Scientific Research Day for Medical, Applied and Basic Sciences, King Khalid University, 5.05.2014, P68.

[30]. U.S. Department of Health and Human Services. Healthy People 2010. 2nd ed. With Understanding and Improving Health and Objectives for Improving Health. 2 vols. Washington, DC: U.S. Government Printing Office; November 2000.

[31]. Khaled Al Akhali, Mohamed Anwar Hammad Ali, Mohammad Asif Ansari1. Evaluation of Prevalence and Pattern of Anemia - A Hospital Based Study in Aseer Province, Kingdom of Saudi Arabia. Journal of Experimental Medical \& Surgical Research. Year XX, Nr. 2 / 2013, Pag. 32 -35.

[32]. U.S. Renal Data System, USRDS 2006 Annual Data Report Atlas of End-Stage Renal Disease in the United States, National Institutes of Health, National Institute of Diabetes and Digestive and Kidney Diseases, Bethesda, MD; 2006.

[33]. Bodenham A, Shelly MP, Park GR. The altered pharmacokinetics and pharmacodynamics of drugs commonly used in critically ill patients. Clin Pharmacokinet. 1988;14:347.

[34]. Winter ME. Basic principles. In: Koda-Kimble MA, Young LY, eds. Basic Clinical Pharmacokinetics. 3rd ed. Vancouver, WA: Applied Therapeutics; 1994:12.

[35]. Faull R, Lee L. Prescribing in renal disease. Aust Prescr 2007;30:17-20. Search PubMed

[36]. Fravel MA, Ernst ME. Management of gout in the older adult. Am J Geriatr Pharmacother 2010;9:271-85. Search PubMed

[37]. Australian Government Department of Veterans' Affairs. Veterans' MATES Therapeutic Brief 21: Revisiting gout management in your veteran patients. 2010.

[38]. Rossi S, editor. Australian Medicines Handbook. Adelaide: Australian Medicines Handbook Ltd, 2012. Search PubMed.

[39]. J Simon Bell, Natalie Blacker, V Tammy LeBlanc, Christopher P Alderman, Adam Phillips, Debra Rowett, et al., Prescribing for older people with chronic renal impairment, Volume 42, No.1, January/February 2013 Pages 24-28

[40]. Randall Faull, Prescribing in renal disease, Australian Prescriber, Volume 30, Number 1, February 2007.

[41]. Livornese LL, et al. Use of antibacterial agents in renal failure. Infectious Disease Clinics of North America. 2004;18:551-79.

[42]. Aronoff GR, Bennett WM, Berns JS, et al, eds. Drug Prescribing in Renal Failure: Dosing Guidelines for Adults and Children. 5th ed. Philadelphia, PA: American College of Physicians; 2007.

[43]. Hammad MA, AL-Akhali KM, Mohammed AT. Evaluation of surgical antibiotic prophylaxisin Aseer area hospitals in Kingdom of Saudi Arabia, JPCS Vol (6), Jan-March 2013.

[44]. Gentry LO. Antimicrobial activity, pharmacokinetics, therapeutic indications and adverse reactions of ceftazidime. Pharmacotherapy 1985;5:254.

[45]. Nicholls PJ. Neurotoxicity of penicillin. J AntimicrobChemother 1980;6:161.

[46]. Nikolaidis P et al. Effect of hemodialysis on ceftazidime pharmacokinetics. Clin Nephrol 1985; 24:142.

[47]. Zaske DE, Cipolle RJ, Rotschafer JC, Solem LD, Mosier NR, Strate RG. Gentamicin pharmacokinetics in 1,640 patients: method for control of serum concentrations. Antimicrobial Agents and Chemotherapy. 1982;21(3):407-411.

[48]. Danish $\mathrm{M}$ et al. Pharmacokinetics of gentamicin and kanamycin during hemodialysis. Antimicrob Agents Chemother 1974;6:841.

[49]. Halpren BA et al. Clearance of gentamicin during hemodialysis: comparison of four artificial kidneys. J Infect Dis 1976;133:627

[50]. Myrna Y. Munar and Harleen Singh. Drug Dosing Adjustments in Patients with Chronic Kidney Disease, American Family Physician, Volume 75, Number 10, May 15, 2007.

[51]. Aronoff GR, Bennett WM, Berns JS, et al. Drug Prescribing in Renal Failure: Dosing Guidelines for Adults and Children. 5th ed. Philadelphia, PA: American College of Physicians; 2007:116.

[52]. Gilbert DN, et al. The Sanford Guide to Antimicrobial Therapy, 38th Edition, 2008.

[53]. Livornese LL, et al. Use of antibacterial agents in renal failure. Infectious Disease Clinics, Volume 23, Issue 4, December 2009, 899 $-924$

[54]. Heintz BH, Matzke GR, Dager WE, "Antimicrobial Dosing Concepts and Recommendations for Critically Ill Adult Patients Receiving Continuous Renal Replacement Therapy or Intermittent Hemodialysis," Pharmacotherapy, 2009, 29(5):562-77. [PubMed 19397464]

[55]. Trotman RL, Williamson JC, Shoemaker DM, et al, "Antibiotic Dosing in Critically Ill Adult Patients Receiving Continuous Renal Replacement Therapy," Clin Infect Dis, 2005, 41(8):1159-66. [PubMed 16163635].

[56]. Heintz, B. H., Matzke, G. R. and Dager, W. E., Antimicrobial Dosing Concepts and Recommendations for Critically Ill Adult Patients Receiving Continuous Renal Replacement Therapy or Intermittent Hemodialysis. Pharmacotherapy: The Journal of Human Pharmacology and Drug Therapy, (2009) 29: 562-577. doi:10.1592/phco.29.5.562.

[57]. Mohamed A. Hammad, Khaled M. AL-Akhali, Asif S. Mohammad, Rahaf M. Alqahtani, Sahar H. Alshhrany, Nada M. Alhassan, Fatma R. Abo Essa. "Prospective Study on Adherence and Pharmacoeconomics of Antibiotic Switching from Intravenous to Oral Route." Journal of Pharmaceutical Research \& Clinical Practice (2015):5(4): 7-13.

[58]. McFadyen ML, Folb PI, Miller R, Keeton GR and Marks IN. Pharmacokinetics of ranitidine in patients with chronic renal failure. Eur J Clin Pharmacol. 1983:25(3):347-51 
[59]. Summary of Product Characteristics - Crestor. Astra Zeneca. Available at: http://emc.medicines.org.uk [Accessed at: 8 - Oct. - 2016]

[60]. Molitch ME. Management of dyslipidemias in patients with diabetes and chronic kidney disease. Clin J Am Soc Nephrol 2006; 1: 1090-9

[61]. Reyes MP et al. Current problems in the treatment of infective endocarditis due to Pseudomonas aeruginosa. Rev Infect Dis $1983 ; 5: 314$

[62]. Perry CM et al. Piperacillin/tazobactam: an updated review of its use in the treatment of bacterial infections. Drugs 1999;57(5):805.

[63]. Aronoff GR et al. The effect of piperacillin dose on elimination kinetics in renal impairment. Eur J ClinPharmacol 1983;24:453.

[64]. Welling PG et al. Pharmacokinetics of piperacillin in subjects with various degrees of renal function. Antimicrob Agents Chemother 1983;23:881.

[65]. Thompson MI et al. Piperacillin pharmacokinetics in subjects with chronic renal failure. Antimicrob Agents Chemother 1981;19:450.

[66]. Giron JA et al. Biliary concentrations of piperacillin in patients undergoing cholecystectomy. Antimicrob Agents Chemother 1981;19:309.

[67]. Austin DJ et al. Vancomycin-resistant enterococci in intensive-care hospital setting; transmission dynamics, persistence, and the impact of infection control programs. ProcNatlAcadSci U S A 1999;96(12):6908.

[68]. Moellering RC et al. Pharmacokinetics of vancomycin in normal subjects and in patients with reduced renal function. Rev Infect Dis 1981;3(Suppl):S230.

[69]. Farber BF, Moellering RC Jr. Retrospective study of the toxicity of preparations of vancomycin from 1974 to 1981 . Antimicrob Agents Chemother 1983;23:138.

[70]. Rodvold, K. A., Blum, R. A., Fischer, J. H., Zokufa, H. Z., Rotschafer, J. C., Crossley, K. B., \& Riff, L. J. Vancomycin pharmacokinetics in patients with various degrees of renal function. Antimicrobial Agents and Chemotherapy, (1988). 32(6), 848852 .

[71]. MacGowan AP. Pharmacodynamics, pharmacokinetics, and therapeutic drug monitoring of glycopeptides. Ther Drug Monit 1998;20(5):473.

[72]. Tan CC et al. Pharmacokinetics of intravenous vancomycin in patients with end-stage renal failure. Ther Drug Monit 1990;12:29. 